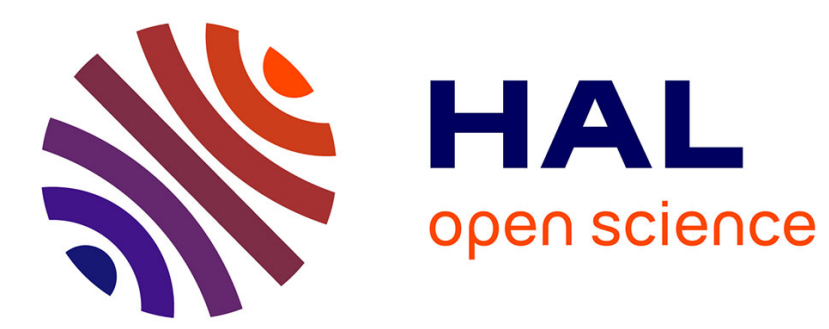

\title{
Improved error bounds for floating-point products and Horner's scheme
}

\author{
Siegfried M. Rump, Florian Bünger, Claude-Pierre Jeannerod
}

\section{To cite this version:}

Siegfried M. Rump, Florian Bünger, Claude-Pierre Jeannerod. Improved error bounds for floatingpoint products and Horner's scheme. BIT Numerical Mathematics, 2016, 56 (1), pp.293 - 307. $10.1007 / \mathrm{s} 10543-015-0555-\mathrm{z}$. hal-01137652

\section{HAL Id: hal-01137652 \\ https://hal.inria.fr/hal-01137652}

Submitted on 31 Mar 2015

HAL is a multi-disciplinary open access archive for the deposit and dissemination of scientific research documents, whether they are published or not. The documents may come from teaching and research institutions in France or abroad, or from public or private research centers.
L'archive ouverte pluridisciplinaire HAL, est destinée au dépôt et à la diffusion de documents scientifiques de niveau recherche, publiés ou non, émanant des établissements d'enseignement et de recherche français ou étrangers, des laboratoires publics ou privés. 


\title{
Improved Error Bounds for Floating-Point Products and Horner's Scheme
}

\author{
Siegfried M. Rump • Florian Bünger • \\ Claude-Pierre Jeannerod
}

Received: date / Accepted: date

\begin{abstract}
Let $\mathbf{u}$ denote the relative rounding error of some floating-point format. Recently it has been shown that for a number of standard Wilkinson-type bounds the typical factors $\gamma_{k}:=k \mathbf{u} /(1-k \mathbf{u})$ can be improved into $k \mathbf{u}$, and that the bounds are valid without restriction on $k$. Problems include summation, dot products and thus matrix multiplication, residual bounds for $L U$ - and Cholesky-decomposition, and triangular system solving by substitution.

In this note we show a similar result for the product $\prod_{i=0}^{k} x_{i}$ of real and/or floatingpoint numbers $x_{i}$, for computation in any order, and for any base $\beta \geqslant 2$. The derived error bounds are valid under a mandatory restriction of $k$. Moreover, we prove a similar bound for Horner's polynomial evaluation scheme.
\end{abstract}

Keywords floating-point product · IEEE 754 standard · Wilkinson type error estimates · Horner scheme

CR Subject Classification $65 \mathrm{G} 50 \cdot 65 \mathrm{~F} 05$

\footnotetext{
Siegfried M. Rump

Institute for Reliable Computing, Hamburg University of Technology, Schwarzenbergstraße 95, Hamburg 21071, Germany, and Faculty of Science and Engineering, Waseda University, 3-4-1 Okubo, Shinjuku-ku, Tokyo 169-8555, Japan (rump@tuhh.de).

Florian Bünger

Institute for Reliable Computing, Hamburg University of Technology, Schwarzenbergstraße 95, Hamburg 21071, Germany (florian.buenger@tuhh.de).

Claude-Pierre Jeannerod

Inria, Laboratoire LIP (CNRS, ENS de Lyon, Inria, UCBL), Université de Lyon, 46 allée d'Italie 69364 Lyon cedex 07, France (claude-pierre. jeannerod@ens-lyon. fr).
} 


\section{Introduction and notation}

Denote by $\mathbb{F}$ a set of floating-point numbers with $p$ digits precision in base $\beta$, and with operations according to IEEE 754 standard [3] in rounding to nearest with any tie breaking rule. Then, $\mathbf{u}:=\frac{1}{2} \beta^{1-p}$ denotes the relative rounding error unit. Throughout the paper we assume that $\beta \geqslant 2$ and $p \geqslant 1$, and that neither overflow nor underflow occurs.

As usual, for $\circ \in\{+,-, \cdot, /\}$ and $a, b \in \mathbb{F}$, the floating-point result of an operation $a \circ b$ is defined to be $\mathrm{fl}(a \circ b)$ for a rounding to nearest $\mathrm{fl}: \mathbb{R} \rightarrow \mathbb{F}$. It follows [2, p. 38] that $|\mathrm{fl}(x)-x| \leqslant \mathbf{u}|x|$ for $x \in \mathbb{R}$, and in particular

$$
|\mathrm{fl}(a \circ b)-(a \circ b)| \leqslant \mathbf{u}|a \circ b|
$$

For matrices $A \in \mathbb{F}^{m \times k}$ and $B \in \mathbb{F}^{k \times n}$, denote by $\widehat{C}$ the floating-point result of the exact product $C:=A B$ computed using (blocked versions of) the classical algorithm, with any ordering for the inner products. A rounding error analysis $\grave{a}$ la Wilkinson then leads typically to $|\widehat{C}-C| \leqslant \gamma_{k}|A \| B|$ with $\gamma_{k}:=\frac{k \mathbf{u}}{1-k \mathbf{u}}=k \mathbf{u}+O\left(\mathbf{u}^{2}\right)$; see for example [2, p. 71]. This standard estimate has been improved in [4] into

$$
|\widehat{C}-C| \leqslant k \mathbf{u}|A||B|
$$

without restriction on the integer $k$ and, in [9], similar improvements have been obtained for the residuals of the computed LU and Cholesky factors as well as for triangular system solutions.

A similar result was recently shown by Graillat, Lefèvre, and Muller [1] for binary arithmetic:

Theorem 1.1 Assume $\beta=2$ and let $x \in \mathbb{F}$ and $k \in \mathbb{N}$ be given. If the power $x^{k+1}$ is computed by successive multiplications by $x$, then, in absence of underflow and overflow, the computed approximation $\widehat{r}$ satisfies

$$
\left|\widehat{r}-x^{k+1}\right| \leqslant k \mathbf{u}\left|x^{k+1}\right| \quad \text { if } \quad k+1 \leqslant \sqrt{2^{1 / 3}-1} \cdot \mathbf{u}^{-1 / 2} .
$$

This improves the classical Wilkinson-type estimate $\left|\widehat{r}-x^{k+1}\right| \leqslant \gamma_{k}\left|x^{k+1}\right|$. They also note that for $k \approx \mathbf{u}^{-1}$ the relative error on $\widehat{r}$ can indeed be larger than $k \mathbf{u}$, thus suggesting that in the case of integer powers, the price to be paid for the refined constant $k \mathbf{u}$ is a necessary restriction on the range of $k$. This is in contrast with bounds like (1.2) and the results in $[4,9]$, where restrictions on $k$ can be avoided.

As Muller [8] mentioned, repeated multiplication may not be the method of choice to evaluate $x^{k+1}$. However, for better methods like binary exponentiation no improvement on the classical constant $\gamma_{k}$ seems to be known.

In this note we generalize Theorem 1.1 to products of real and/or floating-point numbers, to any base, and to any evaluation scheme using $k$ multiplications. Our restriction on $k$ is weaker than the one in (1.3), though of the same order, and we show that it is essentially sharp. 
Theorem 1.2 Let $x_{0}, x_{1}, \ldots, x_{k} \in \mathbb{R}$ be given and suppose that $\ell$ of them are in $\mathbb{F}$. Let also

$$
K:=2 k+1-\ell \quad \text { and } \quad \omega:= \begin{cases}1 & \text { if } \beta \text { is odd }, \\ 2 & \text { if } \beta \text { is even. }\end{cases}
$$

Then, any order of evaluation of the product of $\prod_{i=0}^{k} \mathrm{fl}\left(x_{i}\right)$ produces an approximation $\widehat{r}$ such that, in absence of underflow and overflow,

$$
\widehat{r}-\prod_{i=0}^{k} x_{i}|\leqslant K \mathbf{u}| \prod_{i=0}^{k} x_{i} \mid \quad \text { if } \quad K<\sqrt{\frac{\omega}{\beta}} \mathbf{u}^{-1 / 2} .
$$

In particular, if $\beta=2$ and all the $x_{i}$ are in $\mathbb{F}$, then $(K, \omega)=(k, \beta)$ and (1.5) becomes

$$
\left|\widehat{r}-\prod_{i=0}^{k} x_{i}\right| \leqslant k \mathbf{u}\left|\prod_{i=0}^{k} x_{i}\right| \quad \text { if } \quad k<\mathbf{u}^{-1 / 2} .
$$

For $\beta=2$ and $p \geqslant 4$, the constraint in (1.6) cannot be replaced by $k<12 \mathbf{u}^{-1 / 2}$.

REMARK. Note that for $\beta=2$ and all the $x_{i}$ in $\mathbb{F}$ the restriction $k<\mathbf{u}^{-1 / 2}$ improves on the restriction $k+1 \leqslant \sqrt{2^{1 / 3}-1} \cdot \mathbf{u}^{-1 / 2}=0.509 \ldots \cdot \mathbf{u}^{-1 / 2}$ in (1.3).

The techniques to prove Theorem 1.2 can be used to obtain similar results for other evaluation schemes. As an example we show how to improve the classical factor $\gamma_{2 n}$ for Horner's scheme [2, p. 95].

Theorem 1.3 Let $x, a_{0}, a_{1}, \ldots, a_{n} \in \mathbb{F}$ be given and let $\widehat{r}$ be the approximation to $\sum_{i=0}^{n} a_{i} x^{i}$ produced by Horner's scheme. Then, in absence of underflow and overflow,

$$
\left|\widehat{r}-\sum_{i=0}^{n} a_{i} x^{i}\right| \leqslant 2 n \mathbf{u} \sum_{i=0}^{n}\left|a_{i} x^{i}\right| \quad \text { if } \quad n<\frac{1}{2}\left(\sqrt{\frac{\omega}{\beta}} \mathbf{u}^{-1 / 2}-1\right)
$$

using $\omega$ defined in (1.4).

\section{Products}

We need some preliminaries to prove Theorem 1.2. If some $x_{i}$ is zero, then $\widehat{r}=0$ because no overflow occurs, and the results in Theorem 1.2 are trivial. If all the $x_{i}$ are nonzero, then $\widehat{r} \neq 0$ because, by assumption, no underflow occurs. Furthermore, using $\mathbb{F}=-\mathbb{F}$ and $\mathrm{fl}(-x)=-\mathrm{fl}(x)$, we may henceforth assume without loss of generality that all the $x_{i}$ are positive, so that all the $\widehat{r}_{i}$ are positive as well.

The standard estimate (1.1) can be improved in two ways. First, it is known that

$$
x \in \mathbb{R}: \quad|\mathrm{fl}(x)-x| \leqslant \frac{\mathbf{u}}{1+\mathbf{u}}|x|
$$

and that this bound is sharp; see for example [6, p. 232] and [5]. Second, we use the unit in the first place (ufp): a real number $x$ being given, we set ufp(0) $=0$ and, if 
$x \neq 0, \operatorname{ufp}(x):=\beta^{\left\lfloor\log _{\beta}|x|\right\rfloor}$. Thus, ufp $(x)$ can be thought of as the weight of the first nonzero digit of $x$ in its base $-\beta$ representation. Then,

$$
x \in \mathbb{R}: \quad|\mathrm{fl}(x)-x| \leqslant \mathbf{u} \text { ufp }(x) .
$$

This estimate is sharp as well; for more details, see [10]. Combining (2.1) and (2.2) yields the improved estimate

$$
x \in \mathbb{R} \backslash\{0\}: \quad \mathrm{fl}(x)=x(1+\varepsilon) \quad \text { with } \quad|\varepsilon| \leqslant \min \left[\frac{\mathbf{u}}{1+\mathbf{u}}, \mathbf{u} \frac{\operatorname{ufp}(x)}{|x|}\right] .
$$

In the following we will use

$$
x \in \mathbb{R} \backslash\{0\}: \quad \operatorname{ufp}(x) \leqslant|x|<\beta \operatorname{ufp}(x),
$$

as well as

$$
\begin{array}{rlll}
f \in \mathbb{F} \cap[1, \beta] & \Rightarrow & f=1+2 n \mathbf{u} & \text { with } n \in \mathbb{N}_{0}, \\
f \in \mathbb{F} \cap\left[\beta^{-1}, 1\right] & \Rightarrow & f=1-\frac{2 n}{\beta} \mathbf{u} & \text { with } n \in \mathbb{N}_{0} .
\end{array}
$$

Some notation is necessary to formalize the computation of the floating-point approximation $\widehat{r}$ in (1.5). The evaluation of $\prod_{i=0}^{k} \mathrm{fl}\left(x_{i}\right)$ in any given order by means of $k$ floating-point multiplications is represented by a binary tree $B$ whose $k+1$ leafs correspond to the $\mathrm{fl}\left(x_{i}\right)$ and whose $k$ inner nodes correspond to the multiplications. Thus, $B$ has $2 k+1$ nodes $N_{i}$ in total.

Since the order of evaluation is arbitrary, we may assume without loss of generality that $x_{0}, \ldots, x_{L} \in \mathbb{F}$ with $L:=\ell-1$. The numbering of the nodes shall be such that $N_{i}$ corresponds to $x_{i+L}$ for $i=-L, \ldots, k-L$, and $N_{k-L+1}, \ldots, N_{K}$ are the inner nodes. Moreover, $N_{K}$ shall be the root of $B$.

Each node $N_{i}$ is the root of a tree $B_{i}$ and is identified with the floating-point value $\widehat{r}_{i}=\mathrm{fl}\left(r_{i}\right)$ computed by $B_{i}$. It follows in particular that $\widehat{r}=\widehat{r}_{K}$. More precisely, define $r_{i}:=x_{i+L}$ for $i=-L, \ldots, k-L$ and, by means of a recursive definition, if an inner node $N_{i}, i \in\{k-L+1, \ldots, K\}$, has children $N_{i_{v}}, 1 \leqslant v \leqslant 2$, for which $\widehat{r}_{i_{1}}, \widehat{r}_{i_{2}}$ are already known, define $r_{i}:=\widehat{r}_{i_{1}} \cdot \widehat{r}_{i_{2}}$. Since the $x_{i}$ and $\widehat{r}_{i}$ have been assumed to be positive, the same holds for the $r_{i}$. have

By assumption, $\widehat{r}_{i}=\mathrm{fl}\left(r_{i}\right)=x_{i+L}$ for $i=-L, \ldots, 0$. Moreover, for $i=1, \ldots, K$ we

$$
\widehat{r}_{i}=\mathrm{fl}\left(r_{i}\right)=:\left(1+\varepsilon_{i}\right) r_{i} \quad \text { with } \quad\left|\varepsilon_{i}\right| \leqslant \min \left[\frac{\mathbf{u}}{1+\mathbf{u}}, \mathbf{u} \frac{\mathrm{ufp}\left(r_{i}\right)}{r_{i}}\right]<\mathbf{u} .
$$

For $i \in\{1, \ldots, k-L\}$, the relative errors $\varepsilon_{i}$ correspond to the rounding of $x_{i+L}$ into $\mathrm{fl}\left(x_{i+L}\right)$, while for the remaining indices $i \in\{k-L+1, \ldots, K\}$ they correspond to the $k$ multiplications. This implies $\prod_{i=0}^{k} \mathrm{fl}\left(x_{i}\right)=\prod_{i=1}^{k-L}\left(1+\varepsilon_{i}\right) \cdot \prod_{i=0}^{k} x_{i}$, and therefore

$$
\widehat{r}_{K}-\prod_{i=0}^{k} x_{i}=\left(\prod_{i=1}^{K}\left(1+\varepsilon_{i}\right)-1\right) \cdot \prod_{i=0}^{k} x_{i} \text {. }
$$


Since all factors $x_{i}$ are positive, $(1.5)$ is equivalent to $\left|\prod_{i=1}^{K}\left(1+\varepsilon_{i}\right)-1\right| \leqslant K \mathbf{u}$, and because $\prod_{i=1}^{K}\left(1+\varepsilon_{i}\right) \geqslant(1-\mathbf{u})^{K} \geqslant 1-K \mathbf{u}$ it suffices to prove

$$
\prod_{i=1}^{K}\left(1+\varepsilon_{i}\right) \leqslant 1+K \mathbf{u} .
$$

Hence, we need only upper bounds on the $\varepsilon_{i}$ for the proof of Theorem 1.2.

Furthermore, the lemma below shows that, under weaker assumptions on the maximum $K$, the estimate (1.5) in Theorem 1.2 is true if a single $\varepsilon_{i}$ is not positive, that is, if any of the $k-L$ real $x_{i}$ or any single intermediate product is not rounded upwards. A similar observation was already made in [1, Lemma 3].

Lemma 2.1 With the notation above, in particular (2.6), assume $K \leqslant \sqrt{2} \mathbf{u}^{-1 / 2}$. If there exists an index $i \in\{1, \ldots, K\}$ with $\varepsilon_{i} \leqslant 0$, then (1.5) holds true.

Proof. By (2.6) and (2.8), it suffices to show $Z:=(1+\mathbf{u})^{K-1} \leqslant 1+K \mathbf{u}$. Using $K^{2} \mathbf{u} \leqslant 2$ gives

$$
\ln (Z)=(K-1) \ln (1+\mathbf{u}) \leqslant(K-1) \mathbf{u} \leqslant K \mathbf{u}-\frac{1}{2} K^{2} \mathbf{u}^{2} \leqslant \ln (1+K \mathbf{u}) .
$$

Proof of Theorem 1.2. With the notation above, in particular using (2.6), we have to prove (2.8). For $K \in\{0,1\}$ the assertion is trivial so that henceforth we assume $K \geqslant 2$. By Lemma 2.1 we can also assume that

$$
\varepsilon_{i}>0 \text { for all } i \in\{1, \ldots, K\} .
$$

Let $\varphi \in \mathbb{N}$ be the largest integer satisfying

$$
\varphi<\sqrt{\frac{\omega}{\beta}} \mathbf{u}^{-1 / 2} .
$$

Note that $\varphi \geqslant 2$ because $2 \leqslant K<\sqrt{\omega / \beta} \mathbf{u}^{-1 / 2} \leqslant \varphi+1$. Define $I \subseteq\{1, \ldots, K\}$ to be the index set with

$$
i \in I \quad: \Leftrightarrow \quad \varepsilon_{i}>\frac{\mathbf{u}}{1+\varphi \mathbf{u}} .
$$

The following two properties will be proved for distinct $i, j \in I$ :

a) The nodes $N_{i}$ and $N_{j}$ are not adjacent in the tree $B$.

b) The nodes $N_{i}$ and $N_{j}$ do not have the same parent node in $B$.

Proof of (2.12). In order to derive a contradiction suppose that $N_{i}$ is a child of $N_{j}$. It follows that $r_{j}=\widehat{r}_{i} \widehat{q}$, where $\widehat{q} \in \mathbb{F}$ is a (rounded) $x_{i}$ or some intermediate result. If $\operatorname{ufp}\left(\widehat{r}_{i}\right)=\widehat{r}_{i}$, then $\widehat{r}_{i}$ is a power of $\beta$ and $\varepsilon_{j}=0$ contradicting (2.9), so that (2.6) and $i \in I$ imply

$$
\operatorname{ufp}\left(r_{i}\right)=\operatorname{ufp}\left(\widehat{r}_{i}\right)<r_{i}<(1+\varphi \mathbf{u}) \operatorname{ufp}\left(r_{i}\right) \quad \text { for } \quad i \in I .
$$

Since the second inequality is strict and $1+\varphi \mathbf{u}<1+\sqrt{\mathbf{u}}<\beta$, it follows by (2.5), no matter whether $\varphi$ is odd or even, that

$$
\operatorname{ufp}\left(r_{i}\right)=\operatorname{ufp}\left(\widehat{r}_{i}\right)<\widehat{r}_{i} \leqslant(1+\varphi \mathbf{u}) \operatorname{ufp}\left(r_{i}\right) \quad \text { for } \quad i \in I .
$$


By (2.15) and (2.5) we have

$$
\widehat{r}_{i}=\operatorname{ufp}\left(\widehat{r}_{i}\right)(1+m \mathbf{u}) \quad \text { for even } m \in \mathbb{N} \text { with } 2 \leqslant m \leqslant \varphi .
$$

Hence, $r_{j}=\widehat{r}_{i} \widehat{q},(2.4),(2.16), j \in I$, and (2.14) imply

$$
\frac{R}{1+m \mathbf{u}} \leqslant \frac{r_{j}}{\widehat{r}_{i}}=\widehat{q} \leqslant(1+\varphi \mathbf{u}) R \quad \text { abbreviating } R:=\frac{\operatorname{ufp}\left(r_{j}\right)}{\operatorname{ufp}\left(\widehat{r}_{i}\right)} .
$$

Since $\widehat{q} \in \mathbb{F}, R$ is a power of $\beta$, and $R /(1+m \mathbf{u})>R(1-m \mathbf{u}) \in \mathbb{F},(2.5)$ implies that there exists $v \in \mathbb{Q}$ such that

$$
\widehat{q}=R(1+v \mathbf{u}) \quad \text { and } \quad-m<v \leqslant \varphi .
$$

Moreover, if $v$ is non-negative, then $v$ is a non-negative even integer by (2.5). From (2.18) and (2.16) we get $|v| \leqslant \varphi$. Now $r_{j}=\widehat{r}_{i} \widehat{q},(2.18)$, and (2.16) give

$$
\operatorname{ufp}\left(r_{j}\right) \leqslant r_{j}=\operatorname{ufp}\left(r_{j}\right)\left(1+(m+v) \mathbf{u}+m v \mathbf{u}^{2}\right),
$$

and (2.14) together with $j \in I$ yields

$$
0 \leqslant(m+v) \mathbf{u}+m v \mathbf{u}^{2} \leqslant \varphi \mathbf{u} .
$$

First, assume that $v$ is an even integer. Then, $m+v>0$ is also even by (2.16), so that $1+(m+v) \mathbf{u} \in \mathbb{F}$ and $\left|m v \mathbf{u}^{2}\right| \leqslant \varphi^{2} \mathbf{u}^{2}<\mathbf{u}$ imply $\widehat{r}_{j}=\operatorname{ufp}\left(r_{j}\right)(1+(m+v) \mathbf{u})$ and

$$
\varepsilon_{j}=\frac{\widehat{r}_{j}-r_{j}}{r_{j}}=-\frac{\mathrm{ufp}\left(r_{j}\right) m v \mathbf{u}^{2}}{r_{j}} \leqslant \varphi|v| \mathbf{u}^{2} .
$$

If $v \geqslant 0$, then $\varepsilon_{j} \leqslant 0$, a contradiction. Otherwise, (2.18) and $-v \in \mathbb{N}$ give $|v|=-v \leqslant$ $m-1 \leqslant \varphi-1$, so that $\varphi<\mathbf{u}^{-1 / 2}$ implies

$$
\varphi|v| \mathbf{u}^{2}(1+\varphi \mathbf{u})<\frac{1}{\sqrt{\mathbf{u}}}\left(\frac{1}{\sqrt{\mathbf{u}}}-1\right) \mathbf{u}^{2}(1+\sqrt{\mathbf{u}})=(1-\sqrt{\mathbf{u}}) \mathbf{u}(1+\sqrt{\mathbf{u}}) \leqslant \mathbf{u} .
$$

Hence, $\varepsilon_{j}<\frac{\mathbf{u}}{1+\varphi \mathbf{u}}$ by (2.21), again a contradiction to $j \in I$ by (2.11).

Second, assume that $v$ is not an even integer. Then, (2.18) and (2.5) give $v<0$. Write $v=2 n / \beta=: s+r / \beta$ with $n, s, r \in \mathbb{Z}_{\leqslant 0}$ with $|r|:=(2|n|) \bmod \beta$. Since $2 n$ is even, necessarily

$$
|r| \leqslant\left\{\begin{array}{l}
\beta-2 \text { if } \beta \text { is even, } \\
\beta-1 \text { if } \beta \text { is odd, }
\end{array} \quad \Rightarrow \quad\left|\frac{r}{\beta}\right| \leqslant 1-\frac{\omega}{\beta}\right.
$$

using $\omega$ as in (1.4). In particular, for $\beta=2$ this means $r=0$. Now, (2.19) becomes

$$
r_{j}=\operatorname{ufp}\left(r_{j}\right)(1+(m+s+\delta) \mathbf{u}) \quad \text { with } \quad \delta:=\frac{r}{\beta}+m v \mathbf{u}<0
$$

because $r \leqslant 0$ and $-m \leqslant v<0$. Using (2.22) and (2.10) we obtain

$$
|\delta| \mathbf{u} \leqslant\left(1-\frac{\omega}{\beta}+\varphi^{2} \mathbf{u}\right) \mathbf{u}<\mathbf{u}
$$


If $s$ is odd, then $\delta<0$ and (2.24) yield $\widehat{r}_{j}=\operatorname{ufp}\left(r_{j}\right)(1+(m+s-1) u)$ and $\varepsilon_{j}<0$, a contradiction. If $s$ is even, then $\widehat{r}_{j}=\operatorname{ufp}\left(r_{j}\right)(1+(m+s) \mathbf{u})$ and

$$
\varepsilon_{j}=-\delta \mathbf{u} \frac{\mathrm{ufp}\left(r_{j}\right)}{r_{j}} \leqslant|\delta| \mathbf{u} .
$$

Note that $s$ even implies $r \neq 0$ as $v$ is not an even integer. ${ }^{1}$ By (2.18) we have $-m<$ $v=s+r / \beta$. Since $m, s$ are even integers and $r / \beta<0$, it follows $-m+2 \leqslant s=v-r / \beta$, so that (2.22) yields

$$
|v|=-v \leqslant m-2-\frac{r}{\beta} \leqslant \varphi-1-\frac{\omega}{\beta} .
$$

From (2.25), (2.23), (2.22), (2.16), (2.26), and (2.10) we deduce the final contradiction to $j \in I$ and (2.11):

$$
\frac{\varepsilon_{j}}{\mathbf{u}} \leqslant|\delta| \leqslant 1-\frac{\omega}{\beta}+\varphi\left(\varphi-1-\frac{\omega}{\beta}\right) \mathbf{u}<1-\varphi \mathbf{u}-\frac{\omega}{\beta}+\varphi^{2} \mathbf{u}<1-\varphi \mathbf{u}<\frac{1}{1+\varphi \mathbf{u}}
$$

This finishes the proof of (2.12).

Proof of (2.13). Again, in order to derive a contradiction, assume that $N_{i}$ and $N_{j}$ are the left and right children of an inner node $N_{a}, a \in\{k-L+1, \ldots, K\}$, that is, $r_{a}=\widehat{r}_{i} \widehat{r}_{j}$ and $\widehat{r}_{a}=\mathrm{fl}\left(r_{a}\right)$. Then, like in the proof of (2.12), $i, j \in I$ implies

$$
\begin{aligned}
\operatorname{ufp}\left(r_{i}\right) & =\operatorname{ufp}\left(\widehat{r}_{i}\right)<r_{i}<\widehat{r}_{i}=(1+m \mathbf{u}) \operatorname{ufp}\left(r_{i}\right) \leqslant(1+\varphi \mathbf{u}) \operatorname{ufp}\left(r_{i}\right), \\
\operatorname{ufp}\left(r_{j}\right) & =\operatorname{ufp}\left(\widehat{r}_{j}\right)<r_{j}<\widehat{r}_{j}=(1+n \mathbf{u}) \operatorname{ufp}\left(r_{j}\right) \leqslant(1+\varphi \mathbf{u}) \operatorname{ufp}\left(r_{j}\right)
\end{aligned}
$$

with even $m, n \in \mathbb{N}_{\leqslant \varphi}$. Thus,

$$
r_{a}=\left(1+(m+n) \mathbf{u}+m n \mathbf{u}^{2}\right) \mathrm{ufp}\left(r_{i}\right) \mathrm{ufp}\left(r_{j}\right),
$$

and $(m+n) \mathbf{u} \leqslant 2 \varphi \mathbf{u}<2 \sqrt{\omega / \beta} \mathbf{u}^{1 / 2} \leqslant \frac{2 \omega}{K \beta} \leqslant \frac{2}{K} \leqslant 1$ because $K \geqslant 2$. Moreover, $m+n$ is even and $m n \mathbf{u}^{2} \leqslant \varphi^{2} \mathbf{u}^{2}<\mathbf{u}$. Thus (2.27) yields $\operatorname{ufp}\left(r_{a}\right)=\operatorname{ufp}\left(\widehat{r}_{a}\right)=\operatorname{ufp}\left(r_{i}\right) \operatorname{ufp}\left(r_{j}\right)$, $\widehat{r}_{a}=(1+(m+n) \mathbf{u}) \mathrm{ufp}\left(\widehat{r}_{a}\right)$, and $\varepsilon_{a}=-m n \mathbf{u}^{2} \mathrm{ufp}\left(\widehat{r}_{a}\right) / r_{a}<0$ contradicting (2.9). This finishes the proof of (2.13).

For $I$ consisting of $k^{\prime}$ indices, (2.6) and (2.11) give

$$
\prod_{i=1}^{K}\left(1+\varepsilon_{i}\right) \leqslant\left(1+\frac{\mathbf{u}}{1+\mathbf{u}}\right)^{k^{\prime}}\left(1+\frac{\mathbf{u}}{1+\varphi \mathbf{u}}\right)^{K-k^{\prime}}
$$

Using (2.12) and (2.13) we will show by Lemma 2.2 in Subsection 2.1 that $k^{\prime} \leqslant\left\lfloor\frac{K+1}{2}\right\rfloor$. This implies

$$
\prod_{i=1}^{K}\left(1+\varepsilon_{i}\right) \leqslant\left(1+\frac{\mathbf{u}}{1+\mathbf{u}}\right)^{\left\lfloor\frac{K+1}{2}\right\rfloor}\left(1+\frac{\mathbf{u}}{1+\varphi \mathbf{u}}\right)^{\left\lceil\frac{K-1}{2}\right\rceil} .
$$

1 Thus, for the classical case $\beta=2$ a contradiction to $\{i, j\} \subseteq I$ is already obtained. 
Hence, according to (2.8) and using $\frac{\mathbf{u}}{1+\mathbf{u}} \geqslant \frac{\mathbf{u}}{1+\varphi \mathbf{u}}$, the proof is finished if we show

$$
F(K):=\left(1+\frac{\mathbf{u}}{1+\mathbf{u}}\right)^{\frac{K+1}{2}}\left(1+\frac{\mathbf{u}}{1+\varphi \mathbf{u}}\right)^{\frac{K-1}{2}} \leqslant 1+K \mathbf{u} .
$$

For later use, we do this by proving for real $\psi$ the following stronger statement

$$
G(\psi):=\left(1+\frac{\mathbf{u}}{1+\mathbf{u}}\right)^{\frac{\psi+1}{2}}\left(1+\frac{\mathbf{u}}{1+\psi \mathbf{u}}\right)^{\frac{\psi-3}{2}} \leqslant 1+(\psi-1) \mathbf{u}
$$

provided that $1 \leqslant \psi \leqslant \sqrt{\frac{\omega}{\beta}} \mathbf{u}^{-1 / 2}$. If this is true, then for $1 \leqslant K \leqslant \varphi$ we obtain

$$
F(K) \leqslant G(K)\left(1+\frac{\mathbf{u}}{1+\varphi \mathbf{u}}\right) \leqslant(1+(K-1) \mathbf{u})\left(1+\frac{\mathbf{u}}{1+(K-1) \mathbf{u}}\right)=1+K \mathbf{u}
$$

which is (2.30). A computation yields the Taylor expansion

$$
G(\psi)=1+(\psi-1) \mathbf{u}+\frac{1}{2} G^{\prime \prime}(\xi) \xi^{2} \quad \text { with } \quad G^{\prime \prime}(\xi)=: \alpha N(\xi)
$$

for some $0<\xi<\mathbf{u}$ and

$$
\alpha:=-\frac{(\psi-1)\left(\frac{1+(\psi+1) \xi}{1+\psi \xi}\right)^{\frac{\psi-1}{2}}\left(1+\frac{\xi}{1+\xi}\right)^{\frac{\psi-1}{2}}}{4(1+2 \xi)(1+\xi)^{3}(1+(\psi+1) \xi)^{3}(1+\psi \xi)}<0 .
$$

It suffices to show $N(\xi) \geqslant 0$ for $0<\xi<\mathbf{u}$. Now $N(\xi)=\sum_{\nu=0}^{5} c_{v}$ and $\psi^{2} \mathbf{u} \leqslant 1$ with

$$
\begin{aligned}
c_{0}=60 \xi^{4}+160 \xi^{3}+144 \xi^{2}+48 \xi+4 & >4+48 \xi \\
c_{1}=\left(48 \xi^{5}+192 \xi^{4}+248 \xi^{3}+124 \xi^{2}+20 \xi\right) \psi & >20 \psi \xi+124 \psi \xi^{2} \\
c_{2}=\left(72 \xi^{5}+187 \xi^{4}+140 \xi^{3}+24 \xi^{2}-4 \xi\right) \psi^{2} & >-4 \\
c_{3}=\left(32 \xi^{5}+41 \xi^{4}-8 \xi^{2}\right) \psi^{3} & >-8 \psi \xi \\
c_{4}=\left(8 \xi^{5}+\xi^{4}-4 \xi^{3}\right) \psi^{4} & >-4 \xi \\
c_{5}=-\xi^{4} \psi^{5} & >-\psi \xi^{2} .
\end{aligned}
$$

The series expansions were computed by the Symbolic Math Toolbox of MATLAB [7]. It follows $N(\xi)>0$ for $0<\xi<\mathbf{u}$, and this proves (1.5) and (1.6).

The assertion on possible constraints of $k$ is deferred to the appendix. This finishes the proof of Theorem 1.2.

In the proof of Theorem 1.2 we defined $\varphi$ to be the largest integer less than $\sqrt{\omega / \beta} \mathbf{u}^{-1 / 2}$, which reduces to $\varphi<\mathbf{u}^{-1 / 2}$ for binary arithmetic. Switching from binary arithmetic to another basis requires indeed an adapted definition of $\varphi$. Consider $p:=5$ decimal digits, that is, $\mathbf{u}=0.5 \cdot 10^{-4}$. Then, $\widehat{r}_{i}:=\mathrm{fl}(1.3033 \cdot 0.7697)=1.0032$ and $\widehat{q}:=0.99696$ yield $\widehat{r}_{j}=1.0002$. Moreover, $\varphi=63$ whilst the largest integer less than $\mathbf{u}^{-1 / 2}$ is $\varphi^{\prime}=141$. However, both $\varepsilon_{i}$ and $\varepsilon_{j}$ would satisfy (2.11) if $\varphi$ was replaced by $\varphi^{\prime}$, and indices of adjacent nodes would belong to $I$. 
2.1 A result on colored trees

In (2.29) in the proof of Theorem 1.2 we used the upper bound $\left\lfloor\frac{K+1}{2}\right\rfloor$ for the number $k^{\prime}$ of nodes in the index set $I$. This bound is a consequence of the following lemma.

Lemma 2.2 Let $T$ be a tree with $M$ nodes, each having at most two children. Assume that $C$ nodes of $T$ are colored according to the following rules:

(i) colored nodes are not adjacent;

(ii) each node has at most one colored child.

Then,

$$
C \leqslant \begin{cases}\left\lfloor\frac{M+1}{2}\right\rfloor & \text { if the root of } T \text { is colored, } \\ \left\lfloor\frac{M}{2}\right\rfloor & \text { otherwise. }\end{cases}
$$

Furthermore, these inequalities are sharp for all $M$.

Proof The result is trivial for $M=1$, so assume $M \geqslant 2$ and that the result is true up to $M-1$. The root $R$ of $T$ is then connected to a tree $T_{1}$ and, possibly, also to another tree $T_{2}$ disjoint from $T_{1}$. Let $T_{1}$ have $M_{1}$ nodes, $C_{1}$ of which being colored. Define $M_{2}$ and $C_{2}$ similarly if $T_{2}$ exists, and let $C_{2}=M_{2}=0$ otherwise. Clearly, $M=M_{1}+M_{2}+1$ and $0 \leqslant C_{i} \leqslant M_{i} \leqslant M-1$ for $i=1,2$.

If $R$ is colored, then $C=C_{1}+C_{2}+1$ and (i) implies the root of $T_{1}$ is not colored. Hence, by induction, $C_{1} \leqslant\left\lfloor M_{1} / 2\right\rfloor \leqslant M_{1} / 2$. Similarly, $C_{2} \leqslant M_{2} / 2$, so that

$$
C \leqslant \frac{M_{1}}{2}+\frac{M_{2}}{2}+1=\frac{M+1}{2} \text {. }
$$

If $R$ is not colored, then $C=C_{1}+C_{2}$ and (ii) implies that $R$ has at most one colored child. Hence, for $M_{2}$ either zero or nonzero,

$$
C \leqslant \frac{M_{1}}{2}+\frac{M_{2}}{2}+\frac{1}{2}=\frac{M}{2} .
$$

Since $C$ is an integer, the claimed bounds follow for $M \geqslant 2$. Finally, trees with all internal nodes having exactly one child ("linked lists") and whose colored and uncolored nodes alternate show that the bound is attained for any $M$.

Now, the upper bound for $k^{\prime}$ in the proof of Theorem 1.2 is obtained as follows. First, we construct a tree $T$ by removing from the binary tree $B$ the leafs $N_{-L}, \ldots, N_{0}$ associated with the $\ell$ operands $x_{i}$ already in $\mathbb{F}$. The nodes of $T$ are the nodes $N_{1}, \ldots, N_{K}$ of $B$, and the nodes $N_{i}$ with $i \in I$ are considered as colored. Then, (2.12) and (2.13) imply that $T$ follows the rules (i) and (ii) of Lemma 2.2, so that $|I|=k^{\prime} \leqslant\left\lfloor\frac{K+1}{2}\right\rfloor$.

Optimality of the bounds in Lemma 2.2 is established by linked lists which represent recursive multiplication of floating-point numbers. We note that optimal bounds are attained for other evaluation schemes as well. Examples for all $M$ for trees with colored root are sketched in Figure 2.1; examples with uncolored root follow similarly. 


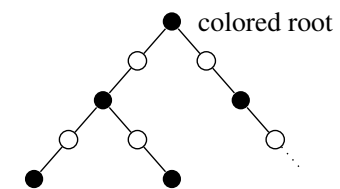

Fig. 2.1 Trees attaining the bound $C=\left\lfloor\frac{M+1}{2}\right\rfloor$ for colored root.

\section{Horner scheme}

Using the techniques of the previous section we prove Theorem 1.3. For $n=0$ the assertion is trivial so that we may assume $n \geqslant 1$. The Horner scheme computes

$\widehat{r}_{0}:=\mathrm{fl}\left(a_{n} x\right) ; \quad \widehat{r}_{i}:=\mathrm{fl}\left(\mathrm{fl}\left(\widehat{r}_{i-1}+a_{n-i}\right) x\right), \quad i=1, \ldots, n-1 ; \quad \widehat{r}=\widehat{r}_{n}:=\mathrm{fl}\left(\widehat{r}_{n-1}+a_{0}\right)$.

For $i=1, \ldots, n$, let the relative error of the $i$-th addition and multiplication be denoted by $\varepsilon_{i}$ and $\varepsilon_{i-1}^{\prime}$, respectively. Then,

$$
\begin{aligned}
& \widehat{r}_{0}=a_{n} x\left(1+\varepsilon_{0}^{\prime}\right), \\
& \widehat{r}_{i}=\left(\widehat{r}_{i-1}+a_{n-i}\right) x\left(1+\varepsilon_{i}\right)\left(1+\varepsilon_{i}^{\prime}\right), \quad i=1, \ldots, n-1, \\
& \widehat{r}=\left(\widehat{r}_{n-1}+a_{0}\right)\left(1+\varepsilon_{n}\right) .
\end{aligned}
$$

For each $i \in\{1, \ldots, n-1\}$ we apply Theorem 1.2 to the product $x_{0} x_{1}$ with $x_{0}:=$ $\widehat{r}_{i-1}+a_{n-i} \in \mathbb{R}$ and $x_{1}:=x \in \mathbb{F}$. Then, $k=1, \ell=1$ and therefore $K=2$, so that (2.29) with the constant $\varphi$ defined in (2.10) yields ${ }^{2}$

$$
\left(1+\varepsilon_{i}\right)\left(1+\varepsilon_{i}^{\prime}\right) \leqslant\left(1+\frac{\mathbf{u}}{1+\mathbf{u}}\right)\left(1+\frac{\mathbf{u}}{1+\varphi \mathbf{u}}\right), \quad i=1, \ldots, n-1 .
$$

Furthermore, (2.6) gives

$$
\left(1+\varepsilon_{0}^{\prime}\right)\left(1+\varepsilon_{n}\right) \leqslant\left(1+\frac{\mathbf{u}}{1+\mathbf{u}}\right)^{2}
$$

From the equalities in (3.1) we deduce that $\widehat{r}=\sum_{i=0}^{n} a_{i}\left(1+\alpha_{i}\right) x^{i}$, where

$$
\begin{aligned}
& 1+\alpha_{n}=\left(1+\varepsilon_{0}^{\prime}\right) \cdot \prod_{j=1}^{n-1}\left(1+\varepsilon_{j}\right)\left(1+\varepsilon_{j}^{\prime}\right) \cdot\left(1+\varepsilon_{n}\right), \\
& 1+\alpha_{i}=\prod_{j=n-i}^{n-1}\left(1+\varepsilon_{j}\right)\left(1+\varepsilon_{j}^{\prime}\right) \cdot\left(1+\varepsilon_{n}\right), \quad i=1, \ldots, n-1, \\
& 1+\alpha_{0}=1+\varepsilon_{n} .
\end{aligned}
$$

Hence, (1.1), (3.2) and (3.3) imply

$$
(1-\mathbf{u})^{2 n} \leqslant 1+\alpha_{n} \leqslant\left(1+\frac{\mathbf{u}}{1+\mathbf{u}}\right)^{n+1}\left(1+\frac{\mathbf{u}}{1+\varphi \mathbf{u}}\right)^{n-1}=: H_{n}
$$

\footnotetext{
${ }^{2}$ In fact, (2.29) is applied to $\left|x_{0}\right|,\left|x_{1}\right|$ because the proof of Theorem 1.2 assumes positive factors.
} 
and, for $i=0, \ldots, n-1$,

$$
(1-\mathbf{u})^{2 i+1} \leqslant 1+\alpha_{i} \leqslant\left(1+\frac{\mathbf{u}}{1+\mathbf{u}}\right)^{i+1}\left(1+\frac{\mathbf{u}}{1+\varphi \mathbf{u}}\right)^{i} .
$$

Then, using $1-2 n \mathbf{u}<(1-\mathbf{u})^{2 n}$, we see that $1-2 n \mathbf{u} \leqslant 1+\alpha_{i} \leqslant H_{n}$ for all $i=0,1, \ldots, n$. The assumption $n<\frac{1}{2}\left(\sqrt{\frac{\omega}{\beta}} \mathbf{u}^{-1 / 2}-1\right)$ implies $2 n+1 \leqslant \varphi$. Thus, (2.31) proves $H_{n} \leqslant G(2 n+1) \leqslant 1+2 n \mathbf{u}$.

We close this note with an application of Theorem 1.2.

\section{Corollary 3.1 (Evaluation of a polynomial given by its roots)}

Given $z, z_{1}, \ldots, z_{n}, a_{n} \in \mathbb{F}$, let $\widehat{r} \in \mathbb{F}$ be a floating-point approximation to

$$
r=a_{n} \prod_{i=1}^{n}\left(z-z_{i}\right)
$$

obtained by first evaluating the $n$ differences and then, in any order, a product of $n+1$ terms. If $n<\frac{1}{2} \sqrt{\frac{\omega}{\beta}} \mathbf{u}^{-1 / 2}$ then, in absence of underflow and overflow,

$$
|\widehat{r}-r| \leqslant 2 n \mathbf{u}|r|
$$

Proof Define $x_{0}:=a_{n} \in \mathbb{F}$ and $x_{i}:=z-z_{i} \in \mathbb{R}$ for $i=1, \ldots, n$. Then, Theorem 1.2 with $k=n, \ell=1, K=2 k+1-\ell=2 n<\sqrt{\frac{\omega}{\beta}} \mathbf{u}^{-1 / 2}$ yields the assertion.

\section{Appendix}

The goal of this appendix is to prove that for $\beta=2$ and $p \geqslant 4$ the constraint $k<\mathbf{u}^{-1 / 2}$ in Theorem 1.2 cannot be replaced by $k<12 \mathbf{u}^{-1 / 2}$. To do that ${ }^{3}$ we construct $x_{0}, x_{1}, x_{2} \in \mathbb{F}$ for given precision $p$ such that $x_{1} x_{2}<1$ and $\operatorname{fl}\left(f l\left(x_{0} x_{1}\right) x_{2}\right)=x_{0}$. Subsequent multiplications by $x_{1} x_{2}$ produce an exponential growth of the rounding error, eventually exceeding $k \mathbf{u}$.

Define $s:=\left\lfloor\mathbf{u}^{-1 / 2}\right\rfloor \in \mathbb{N}$, so that $s=\mathbf{u}^{-1 / 2}-\delta$ with $0 \leqslant \delta<1$. We henceforth assume $p \geqslant 15$ and treat the case $p \leqslant 14$ later. Note that $\beta=2$ and $p \geqslant 15$ imply $s \geqslant 181$. We distinguish two cases.

First, assume $s$ is odd. Set

$$
x_{0}:=1+(2 s+8) \mathbf{u}, \quad x_{1}:=1-(s-4) \mathbf{u}, \quad \text { and } \quad x_{2}:=1+(s-5) \mathbf{u}
$$

so that $x_{i} \in \mathbb{F}$. Then, $x_{0} x_{1}=1+(s+10) \mathbf{u}+\mu_{1} \mathbf{u}$ with $\mu_{1}:=4 \delta \sqrt{\mathbf{u}}+\left(32-2 \delta^{2}\right) \mathbf{u}$, so that $0<\mu_{1}<1$ and $s$ odd imply fl $\left(x_{0} x_{1}\right)=1+(s+11)$ u. Moreover, $\mathrm{fl}\left(x_{0} x_{1}\right) x_{2}=$ $1+(2 s+7) \mathbf{u}+\mu_{2} \mathbf{u}$ with

$$
\mu_{2}:=\sqrt{\mathbf{u}}(6-55 \sqrt{\mathbf{u}}+\Phi \delta) \quad \text { with } \quad \Phi:=(\delta-6) \sqrt{\mathbf{u}}-2 .
$$

\footnotetext{
${ }^{3}$ In [1] long sequences $x_{i} \in \mathbb{F}$ with $\mathrm{fl}\left(\left(\ldots\left(\mathrm{fl}\left(x_{0} x_{1}\right) x_{2}\right) \ldots\right) x_{k}\right)=x_{0}$ are constructed for some precisions.
} 
Now $\Phi<0$ for any value of $\delta$, so that $0<4 \sqrt{\mathbf{u}}-60 \mathbf{u} \leqslant \mu_{2} \leqslant 6 \sqrt{\mathbf{u}}-55 \mathbf{u}<1$. Thus,

$$
\mathrm{fl}\left(\mathrm{fl}\left(x_{0} x_{1}\right) x_{2}\right)=x_{0}
$$

Define a vector $X:=\left[\begin{array}{llll}x_{0} & x & x & \ldots\end{array}\right] \in \mathbb{F}^{2 m+1}$ with $m$ times repeating the row vector $x=\left[\begin{array}{ll}x_{1} & x_{2}\end{array}\right] \in \mathbb{F}^{2}$. Denoting $\widehat{r}_{0}:=x_{0}$ and $\widehat{r}_{i}:=\mathrm{fl}\left(\widehat{r}_{i-1} X_{i}\right)$ for $i \geqslant 1$ yields $\widehat{r}_{2}=v_{0}$. Then, abbreviating $\pi:=x_{1} x_{2}$ and using $\widehat{r}_{2 m}=\widehat{r}_{2}=x_{0}$ gives

$$
\widehat{r}_{2 m}-\prod_{i=0}^{2 m} X_{i}=x_{0}-x_{0} \pi^{m}=\left(\pi^{-m}-1\right) \prod_{i=0}^{2 m} X_{i} \quad \text { for } \quad 1 \leqslant m \in \mathbb{N} .
$$

Now,

$$
\pi=1-(2-(9+2 \delta) \sqrt{\mathbf{u}}) \mathbf{u}-\left(20+9 \delta+\delta^{2}\right) \mathbf{u}^{2}<1-(2-11 \sqrt{\mathbf{u}}) \mathbf{u}=: 1-\gamma \mathbf{u},
$$

and for $m \in \mathbb{N}$,

$$
\pi^{-m}>1+m \gamma \mathbf{u}+\frac{m(m-1)}{2} \gamma^{2} \mathbf{u}^{2}=1+2 m \mathbf{u}+\frac{m \mathbf{u} \sqrt{\mathbf{u}}}{2}\left[(m-1) \gamma^{2} \sqrt{\mathbf{u}}-22\right] .
$$

The assumption $p \geqslant 15$ implies

$$
(6-2 \sqrt{\mathbf{u}}) \gamma^{2}-22=2-272 \sqrt{\mathbf{u}}+(814-242 \sqrt{\mathbf{u}}) \mathbf{u}>2-272 \sqrt{\mathbf{u}}>0,
$$

and therefore

$$
m \geqslant 6 \mathbf{u}^{-1 / 2}-1 \quad \Rightarrow \quad \pi^{-m}>1+2 m \mathbf{u} .
$$

Combining this with (4.2) shows that the error bound in (1.6) is not satisfied for $k=2\left[6 \mathbf{u}^{-1 / 2}-1\right]<12 \mathbf{u}^{-1 / 2}$, and that finishes the first part.

Second, assume $s$ is even and define as before

$$
y_{0}:=1+(2 s+6) \mathbf{u}, \quad y_{1}:=1-(s-3) \mathbf{u}, \quad \text { and } \quad y_{2}:=1+(s-4) \mathbf{u} .
$$

Then, $y_{i} \in \mathbb{F}$. Furthermore, $y_{0} y_{1}=1+(s+7) \mathbf{u}+\mu_{1} \mathbf{u}$ with $\mu_{1}:=4 \delta \sqrt{\mathbf{u}}+\left(18-2 \delta^{2}\right) \mathbf{u}$, so that $0<\mu_{1}<1$ and $s$ even imply fl $\left(y_{0} y_{1}\right)=1+(s+8) \mathbf{u}$. Moreover, fl $\left(y_{0} y_{1}\right) y_{2}=$ $1+(2 s+5) \mathbf{u}+\mu_{2} \mathbf{u}$ with

$$
\mu_{2}:=\sqrt{\mathbf{u}}(4-32 \sqrt{\mathbf{u}}+\Phi \delta) \quad \text { with } \quad \Phi:=(\delta-4) \sqrt{\mathbf{u}}-2 .
$$

As before, $\Phi<0$ for any value of $\delta$. Thus, $0<2 \sqrt{\mathbf{u}}-35 \mathbf{u} \leqslant \mu_{2} \leqslant 4 \sqrt{\mathbf{u}}-32 \mathbf{u}<1$. Hence, similar to (4.1), $\mathrm{fl}\left(\mathrm{fl}\left(y_{0} y_{1}\right) y_{2}\right)=y_{0}$ is again true. Now for the values $y_{1}, y_{2}$ in (4.4) we obtain

$$
y_{1} y_{2}=(1-(s-3) \mathbf{u})(1+(s-4) \mathbf{u})<x_{1} x_{2},
$$

and the result follows as before. Finally, for the cases $4 \leqslant p \leqslant 14$, consider 


\begin{tabular}{rrrrr}
$\mathrm{p}$ & $m_{0}$ & $m_{1}$ & $m_{2}$ & $\mathrm{~F}$ \\
\hline 4 & 2 & -4 & 4 & 9.6 \\
5 & 20 & -3 & 2 & 8.9 \\
6 & 32 & -14 & 16 & 5.8 \\
7 & 28 & -9 & 8 & 6.8 \\
8 & 52 & -39 & 44 & 5.8 \\
9 & 48 & -21 & 20 & 4.6 \\
10 & 140 & -117 & 130 & 5.2 \\
11 & 94 & -43 & 42 & 5.8 \\
12 & 186 & -154 & 158 & 4.0 \\
13 & 184 & -89 & 88 & 4.1 \\
14 & 262 & -125 & 124 & 7.2
\end{tabular}

For precision $p$ define $x_{i}:=1+m_{i} \mathbf{u}$. Then, (4.1) is satisfied, and the error bound in (1.6) is not true for $k<F \mathbf{u}^{-1 / 2}$. This finishes the proof.

We finally mention that it is easy to see that, if $1 \leqslant p \leqslant 2$, then the error bound in (1.6) is satisfied for all $k \in \mathbb{N}$, and if $p=3$, then the minimum value of $k$ for which it is not satisfied is $k=72 \approx 25 \mathbf{u}^{-1 / 2}$.

\section{Summary}

In previous papers, the factor $\gamma_{k}$ has been replaced by $k \mathbf{u}$ in a number of classical error estimates in numerical analysis together with removing the restriction on $k$. We proved that $k \mathbf{u}$ can be used for general products and for the Horner scheme, however, with a mandatory restriction on $k$. So, as by Theorem 1.2, a general principle to replace $\gamma_{k}$ by $k \mathbf{u}$ is necessarily restricted to $k \lesssim \mathbf{u}^{-1 / 2}$.

\section{Acknowledgement}

The authors wish to thank Marko Lange, Vincent Lefèvre, and Jean-Michel Muller for their fruitful and constructive comments on a preliminary version of this note.

\section{References}

1. S. Graillat, V. Lefèvre, and J.-M. Muller. On the maximum relative error when computing integer powers by iterated multiplications in floating-point arithmetic. Research report ensl-00945033, September 2014. Available from https://hal-ens-lyon.archives-ouvertes.fr/ensl-00945033v2.

2. N.J. Higham. Accuracy and Stability of Numerical Algorithms. SIAM Publications, Philadelphia, 2nd edition, 2002.

3. IEEE, New York. ANSI/IEEE 754-2008: IEEE Standard for Floating-Point Arithmetic, 2008.

4. C.-P. Jeannerod and S.M. Rump. Improved error bounds for inner products in floating-point artihmetic. SIAM. J. Matrix Anal. E Appl. (SIMAX), 34(2):338-344, 2013.

5. C.-P. Jeannerod and S.M. Rump. On relative errors of floating-point operations: optimal bounds and applications. Preprint, January 2014

6. D.E. Knuth. The Art of Computer Programming, Volume 2, Seminumerical Algorithms. Third edition, Addison-Wesley, Reading, Massachusetts, 1998.

7. MATLAB. User's Guide, Version 2013b, the MathWorks Inc., 2013. 
8. J.M. Muller. On the maximum relative error when computing iterated integer powers in floating-point arithmetic. INVA conference Tokyo, 2014.

9. S.M. Rump and C.-P. Jeannerod. Improved error bounds for $\mathrm{LU}$ and Cholesky factorizations. SIAM. J. Matrix Anal. E Appl. (SIMAX), 35(2):699-724, 2014.

10. S.M. Rump, T. Ogita, and S. Oishi. Accurate floating-point summation, Part I: Faithful rounding SIAM J. Sci. Comput., 31(1):189-224, 2008. 